\title{
A Rare Variant of Paramesonephric Duct Anomaly - OHVIRA / Herlyn-Werner-Wunderlich Syndrome
}

\author{
Anshul Arora ${ }^{1}$, Akshit Gakhar $^{2}$, Puneet Mittal $^{3}$, Dhaarna Sharma ${ }^{4}$
}

1, 2, 3, 4 Department of Radiology, M.M. Institute of Medical Sciences and Research, Mullana, Ambala, Haryana, India.

\section{INTRODUCTION}

Uterus didelphys with associated obstructed hemivagina and agenesis of the kidney on the same side (OHVIRA) also known as Herlyn-Werner-Wunderlic syndrome is a variant of paramesonephric duct anomaly. Patient usually presents with abdominal pain and dysmenorrhea. We present this case report on OHVIRA syndrome with uterus didelphys from Mullana, Ambala.

Obstructed hemivagina and agenesis of the kidney on the same side (OHVIRA), syndrome is a rare variant of mullerian / paramesonephric duct anomalies. Patient usually presents with abdominal pain and dysmenhorrea. ${ }^{1}$ This syndrome was first described in a young woman by Purslow in 1922 who had a history of progressive pelvic pain and a pelvic mass was felt on palpation, however, she had no menstrual abnormality. ${ }^{2}$ Here a case of a girl aged 13 years is described who had a triad of uterus didelphys, obstructed hemivagina, and agenesis of the kidney on the same side where pelvic ultrasound helped in early diagnosis and then MRI was done for confirmation of findings.

\section{PRESENTATION OF CASE}

A female patient aged 13 years came to outpatient department of gynaecology for evaluation of pelvic pain which radiated to back and right thigh from past 6 months which aggravated during menstruation. Patient did not complaint of any change in her bladder and bowel habits. Patient also denied any menstrual irregularity, vaginal discharge or fever. Patient experienced regular cycles after every 25 - 30 days since her menarche at 12 years. On evaluation she was afebrile with normal vital signs. However, showed mild pallor. Laboratory findings included mildly low haemoglobin of $12.5 \mathrm{gm} \%$, mildly reduced red blood cells of 4.1 million / cumm and mean corpuscular haemoglobin concentration was $30.6 \mathrm{gm} \%$. Her renal function test was also normal with a serum creatinine of $0.54 \mathrm{mg} / \mathrm{dL}$.

Transabdominal ultrasound examination was requested which revealed two horns of uterus. There was also mild endometrial fluid collection in the right horn. The right horn is also communicating inferiorly with a large fluid collection which also shows internal echoes within it. However, both the ovaries appeared normal. Ultrasonography also revealed an absent right kidney in right renal fossa while left kidney appeared normal.

However, the menstrual cycle was normal, a magnetic resonance imaging (MRI) pelvis was advised for further evaluation. MRI revealed complete duplication of uterine body and cervix consistent with uterine didelphys. There was obstruction of right hemivagina due to transverse vaginal septum with associated large haemorrhagic collection measuring approximately $15.1 \times 8.5 \times 9.9 \mathrm{~cm}$ in size in right sided cervix and right hemivagina. The collection was hyperintense on TIW / T2W / T1W SPIR images. Left hemi cervix and hemivagina were compressed due to the collection. Small amount of haemorrhagic fluid was seen to extend into right lower endometrial cavity.

\author{
Corresponding Author: \\ Anshul Arora, \\ Department of Radiology, \\ M.M. Institute of Medical Sciences and \\ Research, Mullana, \\ Ambala, Haryana, India. \\ E-mail: arora.ashu123@gmail.com
}

DOI: $10.14260 /$ jemds/2020/875

How to Cite This Article:

Arora A, Gakhar A, Mittal P, et al. A rare variant of paramesonephric duct anomaly ohvira / herlyn-werner-wunderlich syndrome. J Evolution Med Dent Sci 2020;9(52):4006-4008, $10.14260 /$ jemds/2020/875

Submission 05-09-2020,

Peer Review 02-11-2020,

Acceptance 08-11-2020,

Published 28-12-2020.

Copyright (C) 2020 Anshul Arora et al. This is an open access article distributed under Creative Commons Attribution License [Attribution 4.0 International (CC BY 4.0)] 
Left sided endometrial cavity was normal, endometrial thickness measured around $7 \mathrm{~mm}$ on both sides. Right fallopian tube was also dilated and torturous measuring approximately $17 \mathrm{~mm}$ in diameter and was showing haemorrhagic content. The content was hyperintense on T1 / T2 / TI-SPIR images consistent with haematosalpinx. Both the ovaries were normal. Left ovary also showed a dominant follicle. There was non-visualisation of right kidney in right renal fossa or anywhere else in the abdomen which was consistent with right renal agenesis. Left kidney was normal in size and shape and appeared normal and was normal in position in left renal fossa.
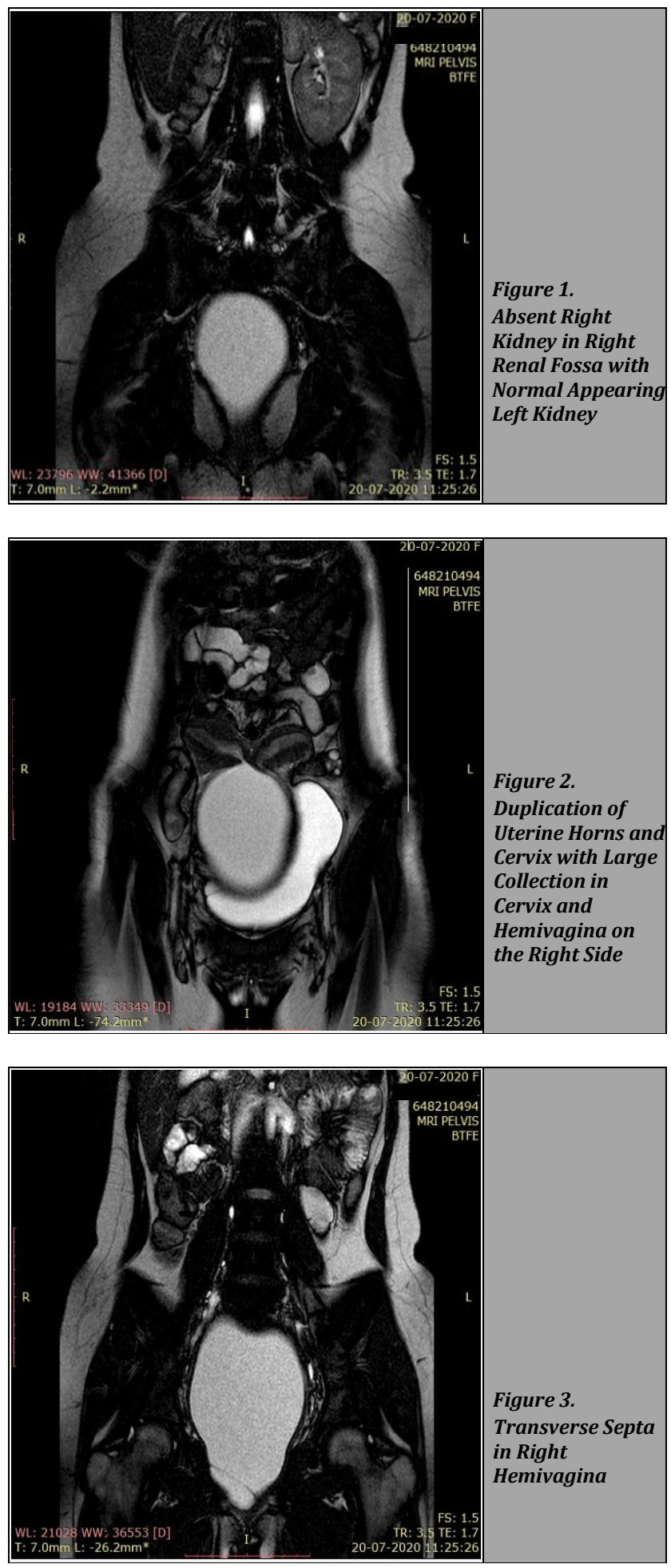
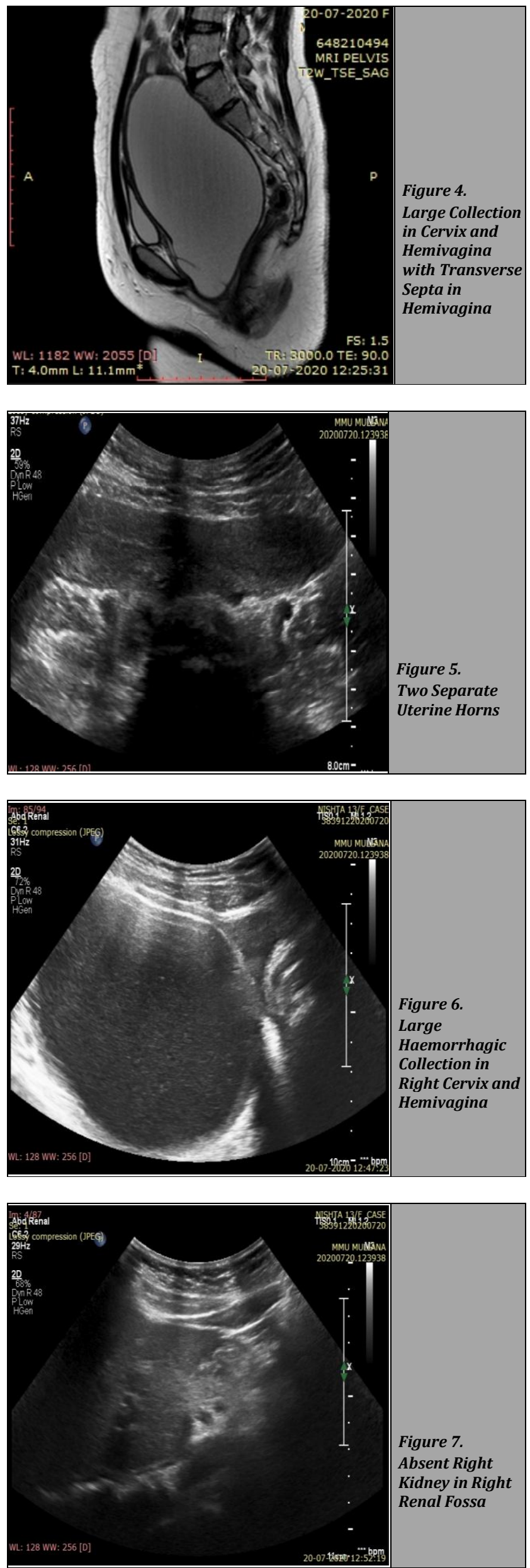


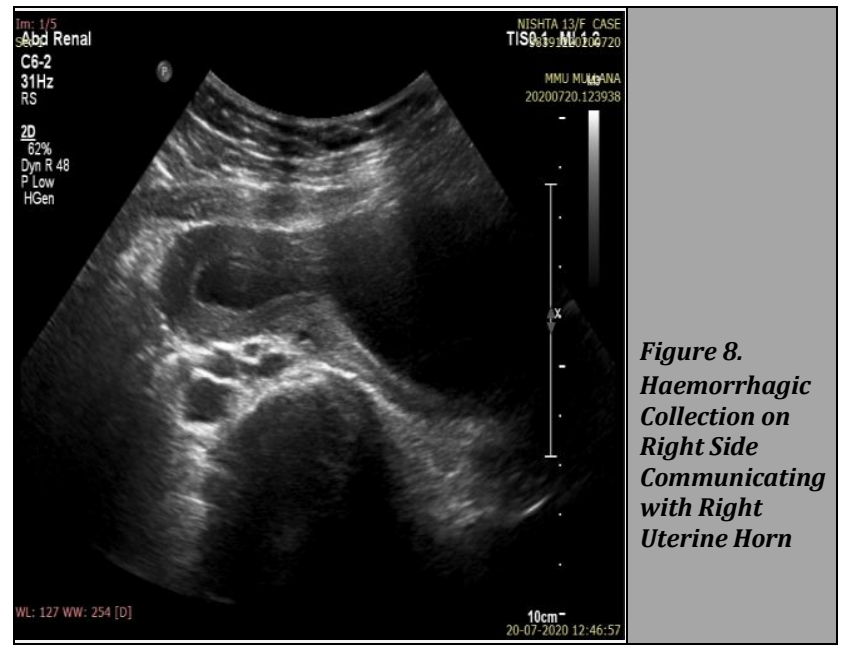

\section{DISCUSSION}

OHVIRA syndrome was first described in a young woman by Purslow in 1922 and later on this was called as HerlynWerner-Wunderlich syndrome, named after the authors who described it in 1970 s. $^{3}$ The incidence rate of this syndrome is $0.1-3 \%{ }^{4}$ This syndrome is a triad of obstructed hemivagina with kidney's agenesis on the same side and uterine didelphys. Its aetiology is not established well. And in order to understand its pathogenesis and association with renal anomalies, we need to be familiar with the embryology of utero-vaginal development. Genetic factors and environmental factors have influence on embryological development. In this syndrome (OHVIRA), the development of the paramesonephric system and metanephros is anamolous. ${ }^{5}$ The urogenital ridge forms the paramesonephric ducts ${ }^{6}$ and later on these paramesonephric ducts embryologically give rise to the uterus and fallopian tubes and cervix along with the upper two-thirds of the vagina. Later on these paramesonephric ducts runs inferiorly (caudad), on the lateral aspect of the mesonephric duct, and at the end the last part comes in the midline on both sides, where both the paramesonephric ducts fuses together and form the uterus and the cervix, and the upper 1 / 3rd of the vagina. ${ }^{6}$ Failure of the fusion of these two paramesonephric ducts can result in mullerian anomalies such as two hemi uteri and hemi cervices which are associated with OHVIRA syndrome. ${ }^{7}$ Absent kidney / ureter on the same side occurs because of the anomalous development of the metanephric diverticulum. ${ }^{8}$ This condition is usually diagnosed around the age of menarche when patient presents with cyclic pain during menstruation along with normal menstrual flow. ${ }^{3}$ Sonography is often the first imaging modality in cases of pelvic pain. Correlation with clinical history of normal menses and haematocolpos should suggest this diagnosis, especially if there is associated renal agenesis. MRI can provide exact detail of the associated anomalies and associated endometriosis, if present. ${ }^{9}$ Surgical manoeuvres are required for the treatment purpose, preferably removal of the obstructing vaginal septum relieving the obstructed hemivagina. ${ }^{10}$ However, in certain cases where maintenance of the integrity of the hymen is primary then vaginal septum resection is done by hysteroscopy with ultrasound guidance.
Rarely, in case of recurrent stenosis unilateral hysterectomy may be considered.

\section{CONCLUSIONS}

OHVIRA syndrome is one of the rare causes of lower abdominal discomfort / pelvic pain in females in young age. Awareness of this condition and appropriate imaging is necessary for correct and timely diagnosis. Less commonly, OHVIRA can present with severe lower abdominal pain / discomfort and findings of cystic adnexal mass with absent (agenesis) kidney on the same side. The diagnosis of OHVIRA / Herlyn-Werner-Wunderlich syndrome is confirmed on MRI. Relieving the obstruction of the genital tract is the primary aim and is usually done surgically.

Financial or other competing interests: None.

Disclosure forms provided by the authors are available with the full text of this article at jemds.com.

\section{REFERENCES}

[1] Piccinini PS, Doski J. Herlyn-Werner-Wunderlich syndrome: a case report. Rev Bras Ginecol Obstet 2015;37(4):192-6.

[2] Purslow CE. A case of unilateral hæmatokolpos, hæmatometra and hæmatosalpinx. BJOG Int J Obstet Gynaecol 1922;29(4):643.

[3] Han B, Herndon CN, Rosen MP, et al. Uterine didelphys associated with obstructed hemivagina and ipsilateral renal anomaly (OHVIRA) syndrome. Radiol Case Rep 2010;5(1):327.

[4] Smith NA, Laufer MR. Obstructed hemivagina and ipsilateral renal anomaly (OHVIRA) syndrome: management and follow-up. Fertil Steril 2007;87(4):91822.

[5] Kimble RMN, Kimble RM. The obstructed hemivagina, ipsilateral renal anomaly, uterus didelphys triad. Fertil Steril 2010;93(4):e15.

[6] Sadler TW. Langman's medical embryology. $12^{\text {th }}$ edn. Philadelphia: Lippincott Williams \& Wilkins 2012.

[7] Acién P, Acien MI. The history of female genital tract malformation classifications and proposal of an updated system. Hum Reprod Update 2011;17(5):693-705.

[8] El-Gohary MA. Uterus didelphys with obstructed hemivagina and ipsilateral renal anomaly (OHVIRA syndrome): a case report. J Pediatr Surg Case Rep 2014;2(9):410-12.

[9] Mandava A, Prabhakar RR, Smitha S. OHVIRA syndrome (obstructed hemivagina and ipsilateral renal anomaly) with uterus didelphys, an unusual presentation. J Pediatr Adolesc Gynecol 2012;25(2):e23-5.

[10] Aveiro AC, Miranda V, Cabral AJ, et al. Herlyn-WernerWunderlich syndrome: a rare cause of pelvic pain in adolescent girls. BMJ Case Rep 2011;2011:bcr0420114147. 